\title{
Multi-line techniques for inference of stellar magnetic fields
}

\author{
Oleg Kochukhov \\ Department of Physics and Astronomy, Uppsala University, Uppsala 75120, Sweden \\ email: oleg.kochukhov@physics.uu.se
}

\begin{abstract}
Spectropolarimetric studies of stellar magnetic fields usually deal with extremely weak line polarisation signatures. The amplitudes of Stokes signals, even in magnetically sensitive spectral lines, are often well below the noise level realistically achievable with the current instrumentation. Consequently, a detection of these polarisation signatures and their meaningful analysis is impossible without combining information from multiple spectral lines. Here I review basic theoretical foundations of the multi-line spectropolarimetric diagnostic methods employed in stellar magnetometry, give examples of their application, and discuss recent efforts to interpret mean polarisation profiles with the help of detailed radiative transfer calculations.
\end{abstract}

Keywords. Polarisation, radiative transfer, stars: activity, stars: magnetic fields

\section{Introduction}

Magnetic fields are believed to play an important role at many stages of stellar formation and evolution (e.g. Mestel 1999). However, many theoretical scenarios involving magnetic fields are lacking observational verification owing to a difficulty of detecting and characterising magnetic fields on the surfaces of stars. From the studies of solar active regions we know that an unambiguous evidence for the presence of magnetic fields is provided by observations of the line splitting and polarisation signatures inside spectral lines, both arising due to the Zeeman effect. But for stars other than the Sun the lack of spatial resolution and much lower photon fluxes tremendously complicate detection of magnetic fields with the Zeeman effect. First, instead of being able to focus on an active region with a strong magnetic field one observes the radiation coming from the entire visible disk of a rotating star. The presence of Doppler shifts due to stellar rotation and the action of other line broadening mechanisms hide Zeeman splitting. Expressing the typical Zeeman splitting in velocity units, $\Delta v_{\mathrm{Z}} \approx 1.4 \times 10^{-3} \lambda g_{\mathrm{eff}} B\left[\mathrm{~km} \mathrm{~s}^{-1} \mathrm{G}^{-1} \mu \mathrm{m}^{-1}\right]$, one can infer that only fields above $\sim 0.5 \mathrm{kG}$ can be potentially detected using magnetically sensitive $\left(g_{\text {eff }}=2-3\right)$ lines provided that the stellar rotational broadening does not exceed a few $\mathrm{kms}^{-1}$ (Andersson et al. 2010; Kochukhov et al. 2013). This kind of diagnostic is therefore limited to slowly rotating stars with very strong fields, such as magnetic Ap stars (Mathys et al. 1997), active M dwarfs (Johns-Krull \& Valenti 1996; Reiners \& Basri 2007), and T Tauri stars (Johns-Krull 2007).

On the other hand, the Zeeman polarisation diagnostic is conceptually easier to apply than the line splitting in intensity spectra since no other processes can lead to similar line polarisation signatures. However, polarisation signals are intrinsically weak. For example, considering the Stokes $V$ profiles in the weak field limit, one can find that the maximum of $|V| / I$ scales with the line-of-sight field component $B_{\mathrm{z}}$ roughly as $2 \times 10^{-4} \lambda g_{\mathrm{eff}} B_{\mathrm{z}}$ $\left[\mathrm{G}^{-1} \mu \mathrm{m}^{-1}\right]$. The amplitude of the Zeeman linear polarisation (Stokes $Q$ and $U$ parameters) is approximately 10 times weaker. In addition, due to its sensitivity to the vector nature of magnetic field, both circular and linear polarisations suffer from a significant 
amount of cancellation when averaged over the stellar surface. Consequently, direct observations of the Zeeman polarisation in individual spectral lines are feasible, again, only for Ap/Bp stars with topologically simple, kG-strength fields (e.g. Silvester et al. 2012; Rusomarov et al. 2013). All other stars, including the sun-like objects, are typically characterised by the disk-averaged fields well below $100 \mathrm{G}$, which could not be directly detected until relatively recently. To this end, enormous recent progress in the studies of stellar magnetism (e.g., see the review by Donati \& Landstreet 2009) came about primarily thanks to the development of wide wavelength coverage spectropolarimeters and application of multi-line signal-enhancing techniques designed to utilise the full information content of spectropolarimetric data.

In this review I describe several multi-line techniques currently used in stellar spectropolarimetry with a focus on the methods that have been widely applied and have led to significant astrophysical results.

\section{The moment technique}

Historically, the moment technique was the first multi-line method systematically applied in the context of stellar magnetism studies. Described in detail by Mathys (1989), this technique considers the low-order moments of the residual Stokes flux profiles $r_{\mathcal{F}_{X}} \equiv\left(\mathcal{F}_{X_{c}}-\mathcal{F}_{X}\right) / \mathcal{F}_{I_{c}}, X=V, Q, U$

$$
R_{X}^{(n)}\left(\lambda_{I}\right)=\frac{1}{W_{\lambda}} \int r_{\mathcal{F}_{X}}\left(\lambda-\lambda_{I}\right)\left(\lambda-\lambda_{I}\right)^{n} d \lambda
$$

where $\lambda_{I}$ is the line's centre-of-gravity, $W_{\lambda}$ is the equivalent width, $\mathcal{F}_{X}$ is the line's flux profile in a given Stokes parameter $X$, and $\mathcal{F}_{X_{c}}$ is the corresponding continuum flux. These Stokes profile moments are in turn related, under the assumption of weak spectral lines and using a simplified treatment (Milne-Eddington atmosphere) of the polarised radiative transfer, to the moments of the magnetic field distribution over the visible stellar surface

$$
\left\langle x^{m} B_{z}^{\ell} B_{j}^{k}\right\rangle=\iint x^{m} B_{z}^{\ell}(x, y) B_{j}^{k}(x, y) \sqrt{1-x^{2}-y^{2}} d x d y .
$$

Here the coordinate system is chosen in such a way that $B_{z}$ is the magnetic field component directed along the line-of-sight and $B_{j}, j=x, y$ denote the components in the plane of the sky $(x, y)$ with the $y$-axis aligned to the stellar rotational axis. In practical applications of the moment technique (Mathys 1991, 1995ab), which were largely limited to strongly magnetic A and B stars, only the first two moments of the Stokes $V$ profiles and the second moment of the Stokes $I$ profiles were considered. The first Stokes $V$ moment allows to measure the mean longitudinal magnetic field $\left\langle B_{z}\right\rangle$ while the second moment characterises the mean field asymmetry or crossover $\left\langle x B_{z}\right\rangle$. Finally, the second Stokes $I$ moment, essentially giving a measure of the differential Zeeman broadening, allows to derive the quantity $\left\langle B^{2}+B_{z}^{2}\right\rangle$ called the mean quadratic magnetic field.

The multi-line aspect of the moment technique is implemented by correlating measurements of individual lines with relevant atomic parameters. Of the three commonly used moments, the mean quadratic field diagnostic is the most uncertain one due to the presence of several competing line broadening processes and occasional profile distortions caused by surface inhomogeneities. This required an ad hoc fine-tuning of the quadratic field determination for individual stars and even for different groups of lines in the same star (Mathys \& Hubrig 2006). 

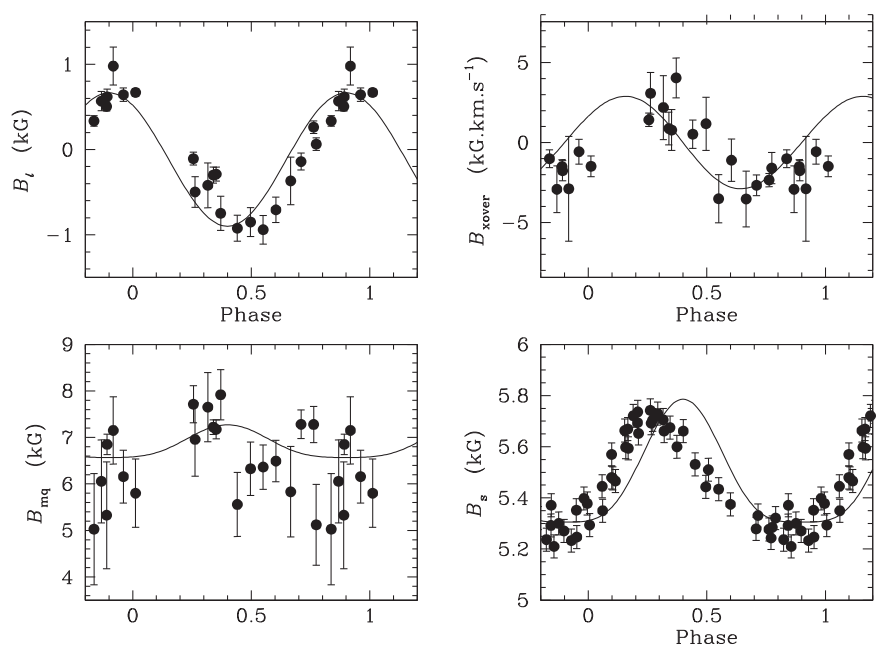

Figure 1. Example of the interpretation of phase curves of the integral magnetic observables (mean longitudinal magnetic field, crossover, mean quadratic field, and the mean field modulus) with a low-order multipolar field model. Here measurements for the Ap star $\beta \mathrm{CrB}$ (symbols) are compared with the prediction (solid line) of the axisymmetric multipolar model geometry discussed by Landstreet \& Mathys (2000).

The measurements of the three Stokes $I$ and $V$ moments can be supplemented by the mean field modulus $\langle B\rangle$ inferred from the splitting of spectral lines in Stokes $I$. The resulting phase curves of the four integral magnetic observables can be interpreted with the help of simple parameterised field topology models, usually involving a combination of the dipole and quadrupole components (Landstreet \& Mathys 2000; Bagnulo et al. 2002). An example of such interpretation is presented in Fig. 1.

This type of modelling has been systematically applied to a few dozen magnetic Ap/Bp stars, allowing to probe the dependence of the global magnetic field characteristics on various stellar parameters. Along these lines it was found that the inclination of the dipolar component with respect to the stellar rotational axis tends to be larger in more rapidly rotating stars (Landstreet \& Mathys 2000) - an observational result still awaiting a meaningful theoretical interpretation.

In several cases multipolar fitting of the integral observables inferred from the Stokes $I$ and $V$ moments has been combined with the interpretation of the broad-band linear polarisation measurements (Bagnulo et al. 2000, 2001). The latter, being particularly sensitive to the transverse field component, yields more robust and complete field topology models.

While the moment technique provided a number of insights into the magnetic properties of intermediate-mass stars with strong, globally organised fields, its usefulness was rather limited for the majority of other stars which have weaker and/or more complex fields. For highly structured surface field geometries, typical of the late-type active stars, the low-order polarisation profile moments are often close to zero despite the presence of significant polarisation signatures and therefore lose their diagnostic value. Moreover, the technical aspects of the application of the moment technique have not been thoroughly validated in the weak field regime. In fact, some claims of the magnetic detections using this technique have been questioned in the literature (Folsom et al. 2010; Shultz et al. 2012; Kochukhov et al. 2013). 


\section{Least-squares deconvolution}

\subsection{Basic principles}

The line addition represents another approach to extracting information from highresolution, wide wavelength coverage polarimetric observations. It is based on the idea that, under certain assumptions, the shapes of spectral lines are self-similar and the line strength depends only on a few parameters such as the central wavelength $\lambda$, residual depth $d$, and some Zeeman sensitivity factor for polarisation profiles. Indeed, in the weak field and weak line limit one can express the local Stokes parameters of the individual lines as scaled profiles $P_{X}(v)$

$$
\begin{aligned}
I_{\mathrm{loc}}(v) \propto & d P_{I}(v) \\
V_{\mathrm{loc}}(v) \propto & \lambda \bar{g} B_{\|} \frac{\partial I_{\mathrm{loc}}}{\partial v}=d \lambda \bar{g} B_{\|} P_{V}(v) \\
Q_{\mathrm{loc}}(v) \propto & \lambda^{2} \bar{G} B_{\perp}^{2} \frac{\partial^{2} I_{\mathrm{loc}}}{\partial v^{2}}=d \lambda^{2} \bar{G} B_{\perp}^{2} P_{Q}(v)
\end{aligned}
$$

where $d$ is the line depth, $\bar{g}$ is the mean Landé factor, $\bar{G}$ is the corresponding parameter characterising the strength of linear polarisation (for triplets $\bar{G}=\bar{g}^{2}$ ), and $B_{\|}, B_{\perp}$ denote the field components parallel and perpendicular to the line of sight. In general, the selfsimilarity of the Stokes profiles is retained through the disk-integration procedure for an arbitrary complex magnetic field geometry. This allows one to introduce disk-integrated mean profiles $Z_{X}(v)$, e.g.

$$
\iint V_{\mathrm{loc}}\left(v-v_{D}\right) d S \propto d \lambda \bar{g} \iint B_{\|} P_{V}\left(v-v_{D}\right) d S \equiv d \lambda \bar{g} Z_{V}(v),
$$

and thus advance a description of the stellar Stokes spectra by a set of superimposed mean profiles, shifted to individual line positions and scaled according to individual weights $w_{i}$

$$
\begin{aligned}
I(v) & =1-\sum_{i} w_{i}^{(I)} Z_{I}\left(v-v_{i}\right), & & w_{i}^{(I)}=d_{i} \\
V(v) & =\sum_{i} w_{i}^{(V)} Z_{V}\left(v-v_{i}\right), & & w_{i}^{(V)}=d_{i} \lambda_{i} \bar{g}_{i} \\
Q(v) & =\sum_{i} w_{i}^{(Q)} Z_{Q}\left(v-v_{i}\right), & & w_{i}^{(Q)}=d_{i} \lambda_{i}^{2} \bar{G}_{i} .
\end{aligned}
$$

As demonstrated by Donati et al. (1997), who were the first to apply this approach to the analysis of Stokes $V$ spectra, Eqs. (3.3) can be inverted with the help of computationally inexpensive matrix operations yielding a high signal-to-noise $(\mathrm{S} / \mathrm{N})$ ratio mean profile for a given observed spectrum, associated error bars, and predefined line mask. Mathematically the resulting mean profile is essentially a cross-correlation function deconvolved from the autocorrelation matrix. Hence the name "least-squares deconvolution" (LSD) frequently used for this technique.

After its introduction for Stokes $V$ by Donati et al. (1997) and extension to linear polarisation by Wade et al. (2000), LSD became de facto standard method of analysing high-resolution night-time spectropolarimetric observations and led to numerous important advances in stellar magnetism research. Those included first quantitative studies of vector magnetic field topologies in late-type active stars (e.g. Donati et al. 2003), detection of ultra-weak fields in normal A-type stars (Lignières et al. 2009), analyses of the magnetic field topologies of solar twins (Petit et al. 2008), low-mass stars (Morin et al. 2008), and accreting T Tauri stars (Donati et al. 2010).

In the most favourable circumstances (e.g. late-type sharp line stars) LSD can be used to combine information from several thousand individual lines, yielding a $\mathrm{S} / \mathrm{N}$ gain of 10 50 and allowing to reach unprecedented polarimetric precision of $\sim 10^{-5}$ and even better. 

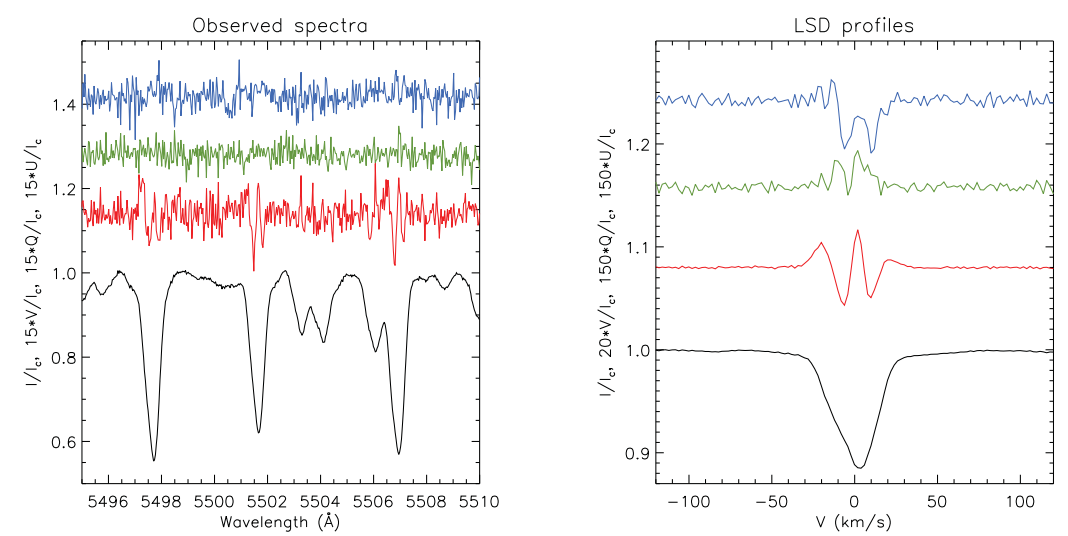

Figure 2. Example of applying the LSD line-addition procedure to the four Stokes parameter spectra of the active cool star II Peg. Left panel: a small segment of spectra centred at the three Fe I lines with large Landé factors. Polarisation signatures are marginally detected in Stokes $V$. Right panel: LSD profiles extracted from the entire optical spectrum of II Peg. Both circular and linear polarisation is clearly detected. Adapted from Rosén et al. (2013). See the on-line edition of this book for a color version of this figure.

As a result, weak polarisation signatures buried in the noise in the original observations become clearly detectable and amenable to detailed interpretation (see Fig. 2).

Several improvements and extensions of the LSD technique were suggested (Sennhauser et al. 2009; Kochukhov et al. 2010), primarily aiming to better account the line-to-line profile differences and improve the treatment of blends. In parallel, alternative multi-line approaches based on the principal component analysis (PCA, Martínez González et al. 2008; Carroll et al. 2012) were considered. Compared to LSD, PCA-based methods may provide some gain in the $\mathrm{S} / \mathrm{N}$ ratio but, at the same time, are more restrictive since they do not include a treatment of overlapping lines and therefore must be applied to unblended spectral features. In general, none of these techniques demonstrated sufficient advantage over LSD to substitute the latter in routine spectropolarimetric applications.

\subsection{Interpretation of $L S D$ profiles}

Depending on the goals of particular studies, LSD Stokes profiles have served different purposes and were interpreted with a varying degree of sophistication. First, one can use mean polarisation signatures merely for the purpose of detecting the presence of a magnetic field. To this end, the $\chi^{2}$ false alarm probability formalism (Donati et al. $1997)$ is widely used. Then, LSD profiles can be employed to compute several integral magnetic observables, similar to the moment technique. In practice, $\left\langle B_{z}\right\rangle$ from Stokes $V$ and the net linear polarisation from Stokes $Q$ and $U$ are the most commonly considered quantities (Silvester et al. 2012). Finally, one can interpret LSD profile shapes in detail, using parametric fitting of the global multipolar field models (e.g. Alecian et al. 2009) or Zeeman Doppler imaging (ZDI) inversions (e.g. Donati et al. 2003).

Evidently, depending on the analysis methodology, the coarse approximations underlying the LSD technique (weak field, weak lines, linear addition of overlapping lines) may result in different types of biases and artefacts. These problems were investigated by Kochukhov et al. (2010), who computed LSD profiles from the synthetic spectra with known parameters and then interpreted these simulated LSD profiles using a number of standard approaches. It was found that, despite the weak field approximation, the Stokes $V$ LSD profiles yield reliable longitudinal magnetic field up to $\left\langle B_{z}\right\rangle \sim 5 \mathrm{kG}$. On the other hand, it was demonstrated that the common assumption of ZDI studies that the LSD 

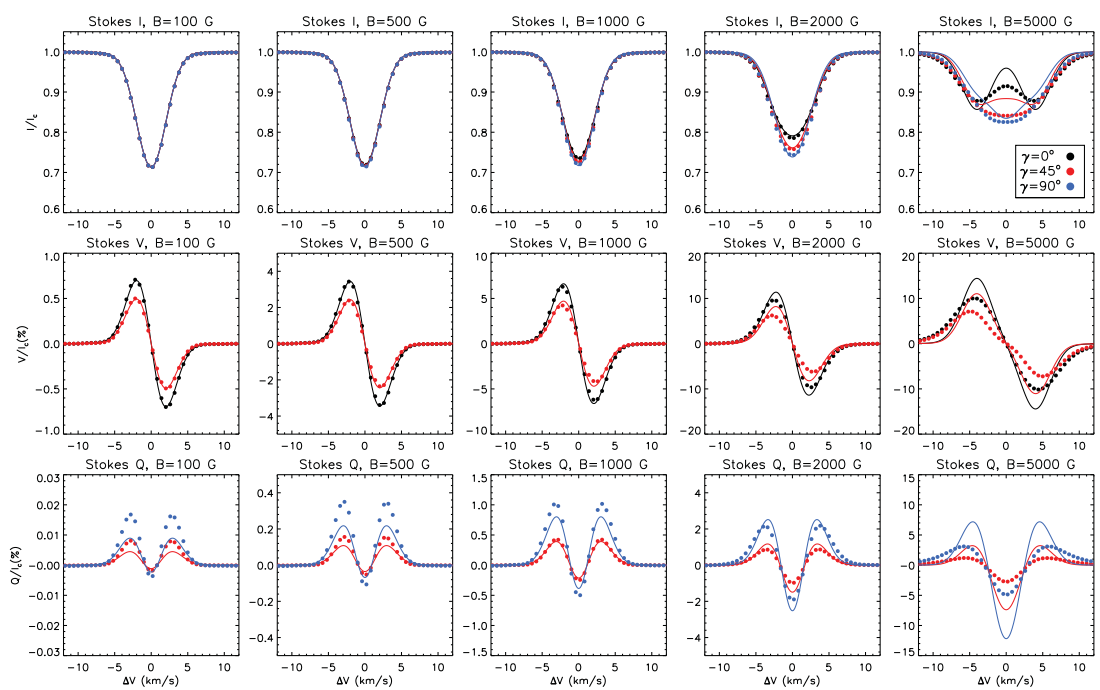

Figure 3. Comparison of the LSD Stokes $I V Q$ profiles obtained from the local synthetic stellar spectra (symbols) with the theoretical line profiles computed for the average line parameters (lines). Different colours illustrate the results for three values of the magnetic field inclination with respect to the line of sight $(0,45$, and 90 degrees). Adapted from Kochukhov et al. (2010). See the on-line edition of this book for a color version of this figure.

profiles behave as a spectral line with mean parameters is applicable only in a limited field strength range. As illustrated by Fig. 3, the synthetic LSD intensity and circular polarisation profiles can be successfully reproduced (after some empirical adjustment of the line strength and width) only up to the field strength of $\sim 2 \mathrm{kG}$. Furthermore, the linear polarisation LSD profiles cannot be reproduced simultaneously with Stokes $V$ at any field strength. The problem with the Stokes $Q$ and $U$ LSD profiles arises due to a diversity of the linear polarisation signatures of real spectral lines and a strong dependence of these signatures on the line strength. Kochukhov et al. (2010) also showed that the response of the Stokes I LSD profile to the variation of chemical abundance or temperature cannot be approximated with a single-line calculation. This implies that DI spot maps inferred from LSD profiles are severely biased compared to the inversion relying on individual lines.

A way to overcome most of the limitations of the LSD-based ZDI and DI studies has been suggested by Kochukhov et al. (2014) and is sketched in Fig. 4. Instead of assigning specific spectral line parameters to the LSD Stokes profiles and modelling them as a single line, one can calculate numerical LSD profiles by applying the line-averaging procedure to the full realistic polarised synthetic spectrum which includes all relevant lines (both those included in the LSD mask and weaker blends). Since the disk integration is a linear operation, these calculations do not need to be repeated for every possible surface magnetic field and spot geometries. Instead, one can precompute the tables of local LSD profiles based on a grid of local Stokes spectra covering a sufficient range of limb angles, field strengths, field inclinations, temperature, etc and then construct diskintegrated synthetic LSD profiles by interpolating within these tables. In this way no specific meaning is attributed to LSD profiles and any approximations involved in their derivation are irrelevant as long as the LSD analysis is performed consistently for the observed and synthetic Stokes spectra.

The new multi-line method of LSD profile modelling was employed to reconstruct selfconsistent chemical abundance and magnetic field maps for the rapidly rotating Ap star 

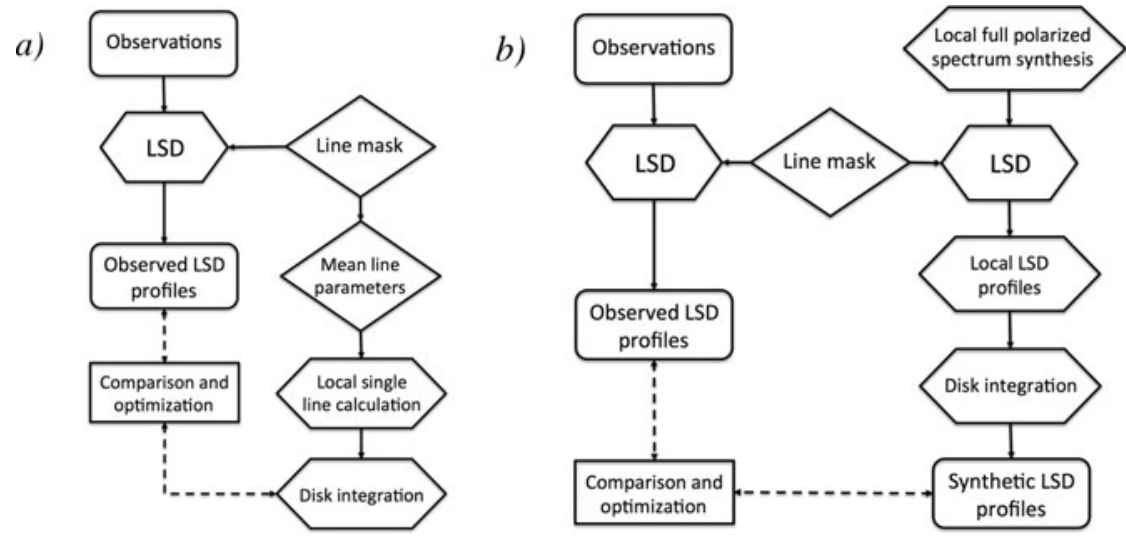

Figure 4. Two methods of interpreting LSD Stokes profiles in Zeeman Doppler imaging: a) traditional single-line approximation and b) realistic polarised radiative transfer approach developed by Kochukhov et al. (2014).
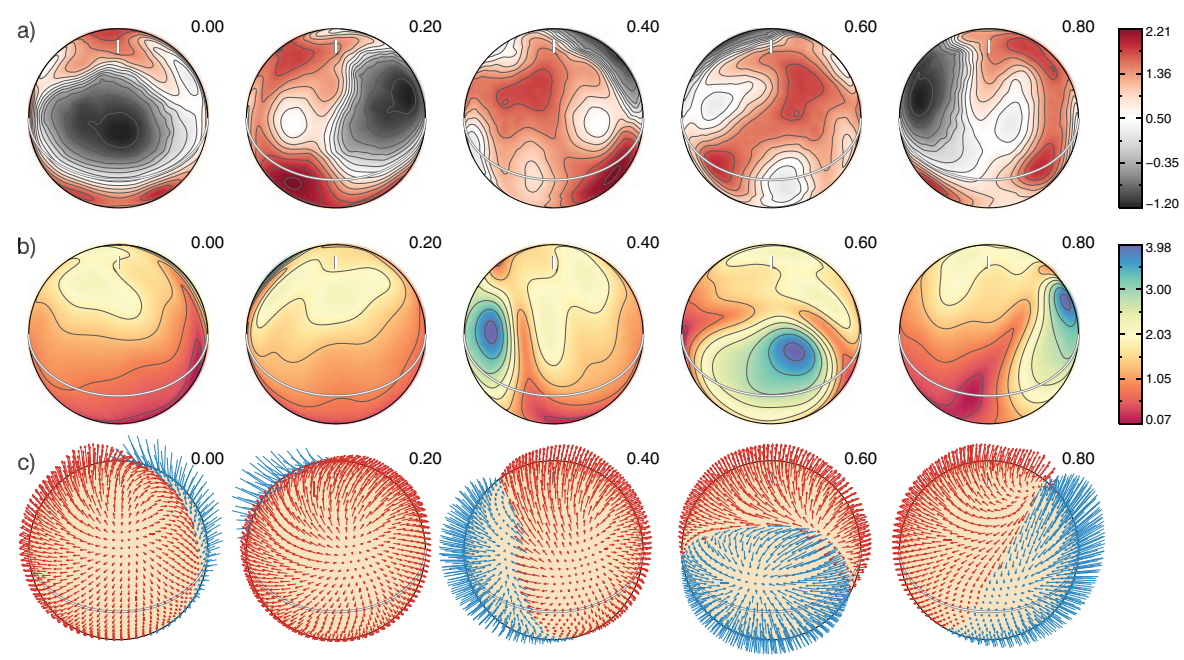

Figure 5. Surface magnetic field and Si abundance distribution of the Ap star CU Vir derived from the Si Stokes $I$ and $V$ LSD profiles (Kochukhov et al. 2014). The spherical plots show the maps of a) Si abundance, b) field modulus, c) field orientation. The thick line and the vertical bar indicate positions of the rotational equator and the pole, respectively. The colour bars give chemical abundance in logarithmic units relative to the Sun and the field strength in $\mathrm{kG}$. See the on-line edition of this book for a color version of this figure.

CU Vir. The results of these inversions, revealing a distorted dipolar field geometry and a high-constant chemical spot distribution, are shown in Fig. 5. In another application Rosén et al. (2015, this meeting) have used theoretical LSD Stokes profile tables to carry out the first ever ZDI mapping of a cool active star in all for Stokes parameters (see Fig. 6). The addition of the LSD Stokes $Q$ and $U$ profiles enabled the magnetic inversion procedure to reconstruct a more detailed and realistic magnetic field configuration. A comparison with the traditional Stokes $V$ inversions from the same data clearly indicates that ZDI with only circular polarisation misses much of the field complexity and tends to significantly underestimate the field strength. 


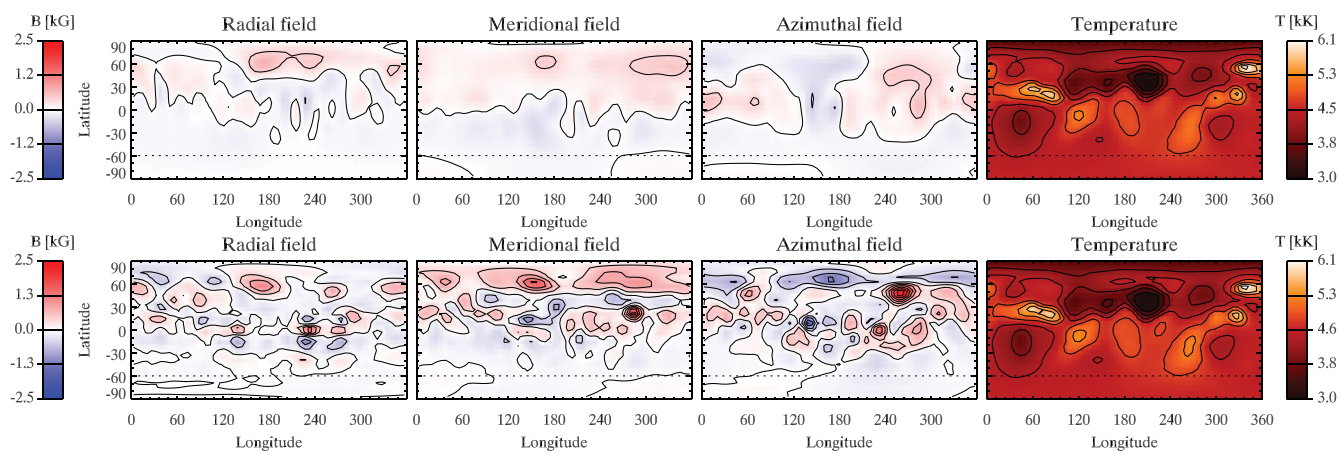

Figure 6. Surface magnetic field geometry and temperature distribution recovered for the RS CVn star II Peg (Rosén et al. 2015). The top row corresponds to the Stokes $I V$ inversion. The bottom row presents results of the Stokes $I Q U V$ reconstruction. In both cases full radiative transfer calculations were used to model the observed LSD polarisation profiles. See the on-line edition of this book for a color version of this figure.

\section{Conclusions}

To summarise, the multi-line approaches in stellar magnetism studies have evolved from application of the moment technique to strongly magnetic Ap/Bp stars to a universal usage of least-squares deconvolution. LSD has proven to be a very useful and robust method of the magnetic field detection and characterisation. Its numerous assumptions, although certainly restrictive, are detrimental only in the context of detailed line profile modelling, e.g. by ZDI. Even in this case unphysical simplifications can, in principle, be avoided with the help of multi-line polarised radiative transfer calculations.

\section{References}

Alecian, E., Wade, G. A., Catala, C., et al. 2009, MNRAS 400, 354

Anderson, R. I., Reiners, A., \& Solanki, S. K. 2010, A\&\&A 522, A81

Bagnulo, S., Landolfi, M., Mathys, G., et al. 2000, A\&A 358, 929

Bagnulo, S., Wade, G. A., Donati, J.-F., et al. 2001, A\&SA 369, 889

Bagnulo, S., Landi Degl'Innocenti, M., Landolfi, M., et al. 2002, A\&A 394, 1023

Carroll, T. A., Strassmeier, K. G., Rice, J. B., et al. 2012, A 6 A 548, A95

Donati, J.-F., Semel, M., Carter, B. D., et al. 1997, MNRAS 291, 658

Donati, J.-F., Cameron, A. C., Semel, M., et al. 2003, MNRAS 345, 1145

Donati, J.-F. \& Landstreet, J. D. 2009, ARA\&A 47, 333

Donati, J.-F., Skelly, M. B., Bouvier, J., et al. 2010, MNRAS 409, 1347

Folsom, C. P., Kochukhov, O., Wade, G. A., et al. 2010, MNRAS 407, 2383

Johns-Krull, C. M. 2007, ApJ 664, 975

Johns-Krull, C. M. \& Valenti, J. A. 1996, ApJ 459, L95

Kochukhov, O., Makaganiuk, V., \& Piskunov, N. 2010, A\& A 524, 5

Kochukhov, O., Makaganiuk, V., Piskunov, N., et al. 2013, A\&SA 554, A61

Kochukhov, O., Lüftinger, T., Neiner, C., et al. 2014, A\&SA 565, 83

Landstreet, J. D. \& Mathys, G. 2000, A\& $A$ 359, 213

Lignières, F., Petit, P., Böhm, T., et al. 2009, A $\& A$ 500, L41

Martínez González, M. J., Asensio Ramos, A., Carroll, T. A., et al. 2008, A $\& A$ 486, 637

Mathys, G. 1989, Fundamentals of Cosmic Physics 13, 143

Mathys, G. 1991, A\&SA 89, 121

Mathys, G. 1995a, $A \mathscr{E} A$ 293, 733

Mathys, G. 1995b, A\&\& 293, 746

Mathys, G., Hubrig, S., Landstreet, J. D., et al. 1997, A\&SAS 123, 353 
Mathys, G. \& Hubrig, S. 2006, A\& A 453, 699

Mestel, L. 1999, Stellar magnetism, Clarendon, Oxford

Morin, J., Donati, J.-F., Petit, P., et al. 2008, MNRAS 390, 567

Petit, P., Dintrans, B., Solanki, S. K., et al. 2008, MNRAS 388, 80

Reiners, A. \& Basri, G. 2007, ApJ 656, 1121

Rosén, L., Kochukhov, O., \& Wade, G. A. 2013, MNRAS 436, L10

Rosén, L., Kochukhov, O., \& Wade, G. A. 2015, ApJ submitted

Rusomarov, N., Kochukhov, O., Piskunov, N., et al. 2013, A\&A 558, A8

Sennhauser, C., Berdyugina, S. V., \& Fluri, D. M. 2009, A\&A 507, 1711

Silvester, J., Wade, G. A., Kochukhov, O., et al. 2012, MNRAS 426, 1003

Shultz, M., Wade, G. A., Grunhut, J., et al. 2012, ApJ 750, 2

Wade, G. A., Donati, J.-F., Landstreet, J. D., et al. 2000, MNRAS 313, 823 